\title{
Substitution of dietary saturated fatty acids with either monounsaturated or $n-6$ polyunsaturated fatty acids improves the plasma lipid profile in those at risk from CVD: results from the DIVAS study
}

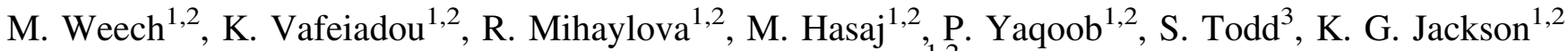 \\ and J. A. Lovegrove ${ }^{1,2}$ \\ ${ }^{1}$ Hugh Sinclair Unit of Human Nutrition, ${ }^{2}$ Institute of Cardiovascular and Metabolic Research (ICMR) and ${ }^{3}$ Department of \\ Mathematics and Statistics, University of Reading, Reading, RG6 6AP, UK
}

Current dietary guidelines have focused on the reduction of dietary saturated fatty acid (SFA) intake as a means of reducing cardiovascular disease risk (CVD). Questions still remain as to whether monounsaturated (MUFA) or $n-6$ polyunsaturated (n-6 PUFA) fatty acids are the optimal macronutrient to replace dietary SFA. The aim of DIVAS (Dietary Intervention and VAscular function Study; ClinicalTrials.gov NCT01478958) was to determine the impact of substitution of dietary SFA with either MUFA or $n-6$ PUFA on plasma lipids and markers of insulin resistance in adults at an increased risk of developing CVD.

Men and women ( $n=195$, mean (SD) age 44 (10) years and BMI $26.9(4.0) \mathrm{kg} / \mathrm{m}^{2}$ ) participated in a 16-week, parallel, randomised, controlled, single-blinded intervention study. Volunteers were randomly assigned (minimised by gender, age, BMI and CVD risk score) to one of three isoenergetic diets: SFA-rich (target composition: $36 \%$ of total energy (\%E) as total fat, $17 \% \mathrm{E}$ SFA, $11 \% \mathrm{E}$ MUFA, $4 \% \mathrm{E} n-6$ PUFA), MUFA-rich (36\% E total fat, 9\%E SFA, 19\%E MUFA, 4\%E $n$-6 PUFA), or $n$-6 PUFA-rich (36\% E total fat, $9 \% \mathrm{E}$ SFA, $13 \% \mathrm{E}$ MUFA, $10 \% \mathrm{E} n-6$ PUFA). For the successful delivery of the dietary targets, a flexible dietary exchange model was developed in which exchangeable fats in the habitual diet were replaced by study foods (spreads, oils, snacks) with a specific fatty acid profile. Fasting blood total cholesterol (TC), high-density lipoprotein cholesterol (HDL-C), triacylglycerol (TAG), glucose, insulin and non-esterified fatty acids (NEFA) concentrations were measured at baseline and week 16. Low-density lipoprotein cholesterol (LDL-C) concentrations were estimated by using the Friedewald formula ${ }^{(1)}$. Homeostasis model assessment (HOMA) was calculated as an index of insulin resistance ${ }^{(2)}$.

There was a significant effect of diet on fasting TC, LDL-C and TC:HDL-C ratio with significantly lower concentrations observed after the MUFA-and PUFA-diets compared with the SFA-rich diet. No differences were evident between the PUFA-rich and MUFA-rich diets. Dietary fatty acid intake did not significantly influence HOMA, blood glucose, insulin or NEFA levels (data not shown).

\begin{tabular}{|c|c|c|c|c|c|c|c|}
\hline \multirow[b]{3}{*}{ Lipids $(\mathrm{mmol} / \mathrm{l})$} & \multicolumn{6}{|c|}{ Change in lipids and TC:HDL-C ratio following the dietary fat manipulation (wk16-wk0) } & \multirow[b]{3}{*}{$P^{l}$} \\
\hline & \multicolumn{2}{|c|}{ SFA rich diet $(n=65)$} & \multicolumn{2}{|c|}{ MUFA rich diet $(n=64)$} & \multicolumn{2}{|c|}{$n$-6 PUFA rich diet $(n=65)$} & \\
\hline & Mean & SEM & Mean & SEM & Mean & SEM & \\
\hline TC & 0.36 & 0.08 & $-0.08 *$ & 0.09 & $-0.10 *$ & 0.09 & 0.001 \\
\hline HDL-C & 0.06 & 0.02 & 0.01 & 0.02 & 0.05 & 0.03 & 0.448 \\
\hline LDL-C & 0.30 & 0.07 & $-0.09 *$ & 0.08 & $-0.15^{*}$ & 0.07 & $<0.001$ \\
\hline TC:HDL-C & 0.13 & 0.06 & $-0.10 *$ & 0.06 & $-0.18 *$ & 0.06 & 0.001 \\
\hline TAG & 0.03 & 0.05 & 0.01 & 0.05 & -0.03 & 0.06 & 0.595 \\
\hline
\end{tabular}

${ }^{1}$ Derived by Univariate Analysis for between diet comparison with baseline, BMI and age as covariates followed by the LSD post-hoc test; $*$ significantly different from the SFArich diet $(P<0.05)$

In conclusion, replacement of dietary SFA for 16 weeks with either MUFA or $n-6$ PUFA resulted in a shift towards a beneficial lipid profile in men and women at increased CVD risk.

The DIVAS study was supported by the Department of Health and the UK Food Standards Agency (N02044).

1. Friedewald WT, Levy RI \& Fredrickson DS (1972) Clin Chem 18, 499-502.

2. Matthews DR, Hosker JP, Rudenski AS et al. (1985) Diabetologia 28, 412-419. 